$\overline{A E E T}$

ASOCIACIÓN ESPAÑOLA DE ECOLOGÍA TERRESTRE

\title{
Las invasiones biológicas y su impacto en los ecosistemas
}

\author{
A. Alonso ${ }^{1, *}$, P. Castro-Díez ${ }^{1}$ \\ (1) Departamento de Ciencias de la Vida, Unidad Docente de Ecología, Universidad de Alcalá, Crta. N-II Km 33,6 Campus Universitario, Edificio Biología, \\ 28805 Alcalá de Henares, Madrid, España \\ *Autor de correspondencia: A. Alonso [aafernandez1976@yahoo.es]
}

> Recibido el 24 de marzo de 2015 - Aceptado el 25 de marzo de 2015

Alonso, A., Castro-Díez, P. 2015. Las invasiones biológicas y su impacto en los ecosistemas. Ecosistemas 24(1):1-3. Doi.: 10.7818/ECOS.2015.24-1.01

Durante las últimas décadas el ser humano ha roto muchas barreras geográficas que han permitido la dispersión de numerosas especies de seres vivos. Algunas de estas especies se han convertido en invasoras, causando graves impactos en la estructura y funcionamiento de los ecosistemas invadidos. Por tanto las invasiones biológicas constituyen una parte importante, aunque menos conocida, del llamado "cambio global" que sufre nuestro planeta, contribuyendo de forma considerable a la pérdida de biodiversidad y de los servicios de los ecosistemas. Algunos autores han llegado a llamar a este proceso la "macdonaldización de la biosfera" en clara similitud con la globalización económica que sufren nuestras sociedades. Por ejemplo, en la base de datos de Delivering Alien Invasive Species Inventories for Europe aparecen un total de 12122 especies exóticas presentes en Europa. Algunas de estas especies pueden causar notables pérdidas económicas directas, por ejemplo inutilizando infraestructuras; otras especies representan una amenaza para la salud humana, transmitiendo enfermedades, produciendo biotoxinas o causando alergias (Mazza et al. 2014); otras reducen el valor de los servicios que prestan los ecosistemas, mermando su capacidad para proveer recursos, para regular ciclos de agua y nutrientes, o simplemente reduciendo su valor estético (Charles y Dukes 2007). En Estados Unidos se ha estimado que las especies exóticas suponen un coste de unos 120000 millones de dólares al año en EEUU ( $\mathrm{Pi}$ mentel 2005). En España el coste directo de las medidas de gestión de especies invasoras durante la primera década del presente siglo se ha estimado en unos 50.5 millones de euros (Andreu et al. 2009). Esto da una idea de la magnitud del problema ecológico y económico al que se enfrenta la humanidad.

En la década de los 50 del pasado siglo el ecólogo británico Charles S. Elton sentó las bases de la ecología de las invasiones biológicas en su famoso libro The ecology of invasions by plants and animals (Elton 1958). Este autor advirtió de los impactos que las especies exóticas podían causar en los ecosistemas, aportando ejemplos tan graves como la destrucción de numerosas poblaciones de cangrejos europeos por un patógeno fúngico desde los años 70 del siglo XIX. Tres décadas más tarde, un estudio sobre el impacto del arbusto exótico Myrica faya en el ciclo del nitrógeno en las islas de Hawái tuvo una gran repercusión científica. Vitousek y Walker (1989) estimaron que la invasión de esta planta, fijadora de nitrógeno atmosférico, había cuadruplicado la entrada de nitrógeno en los suelos volcánicos oligótrofos de estas islas, facilitando a su vez la invasión de otras plantas exóticas. El impacto de este y otros estudios hizo que, poco a poco, la comunidad científica haya tomado conciencia de la importancia de este problema, aportando cada vez más evidencias de impactos de las especies exóticas invasoras en diferentes ecosistemas del planeta. El creciente interés de los ecólogos en las causas y consecuencias de las invasiones biológicas se ha reflejado en el auge de las publicaciones científicas en las últimas décadas (Fig. 1). Además han aparecido revistas internacionales especializadas, como Biological Invasions (1999) o Aquatic Invasions (2006), ambas incluidas en el Journal Citation Report. También hay que remarcar que este tema es cada vez más tratado en revistas generales de ecología y de ciencias ambientales. Por último, la prensa también está haciendo eco, cada vez con más frecuencia, de los casos de invasión con impactos más notables, como el del mejillón cebra (Dreissena polimorpha) en la cuenca del Ebro, el mapache (Procyon lotor) en el Parque Regional del Sureste (Madrid), el mosquito tigre (Aedes albopictus) en la costa mediterránea o la avispa asiática (Vespa vetulina) en el norte de España.

El presente monográfico muestra doce trabajos -tanto de investigación como de revisión- sobre el impacto de las especies exóticas invasoras en diferentes ecosistemas (acuáticos y terrestres), para diferentes grupos taxonómicos y en diferentes zonas geográficas del mundo (América y Europa). Cinco de estos trabajos se centran en los ecosistemas acuáticos epicontinentales, estos ecosistemas se encuentran entre los más afectados por el impacto de las especies exóticas invasoras. El artículo de García-Berthou et al. (2015) revisa el conocimiento sobre el impacto que causan los peces exóticos introducidos en los ecosistemas acuáticos continentales de la península ibérica. Asimismo, muestra cómo esas especies impactan en todos los niveles de organización del ecosistema, desde los más bajos (por ejemplo induciendo hibridación entre especies o alterando el flujo genético entre poblaciones) hasta los más altos (por ejemplo, modificando la diversidad). Estos autores muestran cómo algunas especies basan su éxito en los drásticos cambios de dieta que sufren a lo largo de su desarrollo ontogenético y en su gran plasticidad trófica, que les permite minimizar la competencia con las especies nativas. Tal es el caso de la perca americana (Micropterus salmoides), una de las peores especies invasoras a nivel mundial que el hombre ha favorecido para su uso 


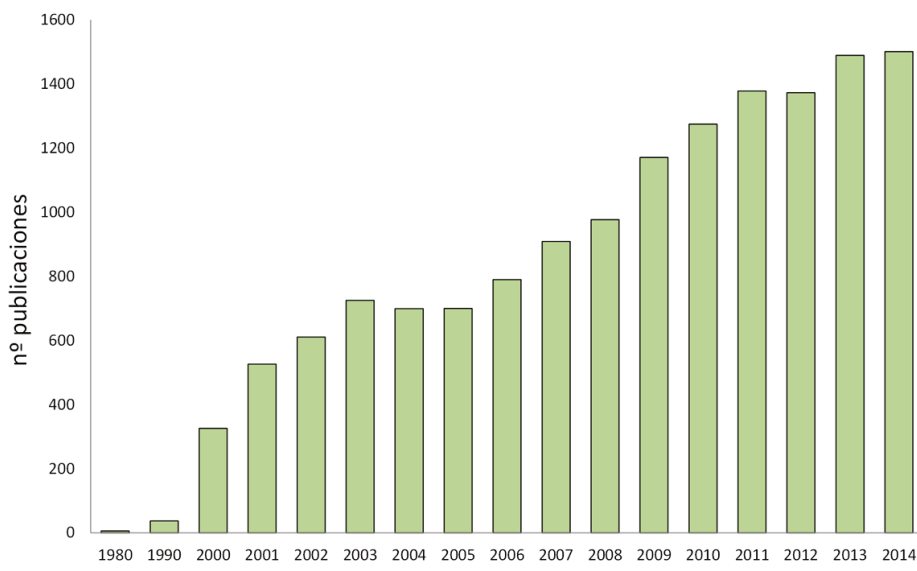

Año

Figura 1. Número de artículos científicos publicados por año en revistas internacionales incluidas en el Journal Citation Report relacionadas con las especies exóticas invasoras y la ecología. Se ha empleado el buscador Web of Science con la siguiente combinación de palabras claves en el área TEMA: ("biological invasions" or "exotic species" or "invasive species" or "non-native species") AND (Ecology or Ecosystem*).

Figure 1. Per-year number of scientific articles published in international journals included in the Journal Citation Report on invasive exotic species and ecology is presented. The Web of Science has been used as searcher with the following searching formula: TOPIC: ("biological invasions" or "exotic species" or "invasive species" or "non-native species") AND (Ecology or Ecosystem*).

en la pesca deportiva. Otro de los trabajos presentados analiza el impacto de los salmónidos en los ecosistemas acuáticos epicontinentales de Chile (Habit et al. 2015). De las 72 especies de peces continentales chilenas 27 han sido introducidas; el $43 \%$ de estas últimas pertenece a la familia Salmonidae, con dos especies distribuidas por todo el país: la trucha común (Salmo trutta) y la trucha arcoíris (Oncorhynchus mykiss). Estos autores muestran que una parte importante del impacto causado por los salmónidos sobre los peces nativos de la Patagonia chilena se debe a las nuevas relaciones tróficas que generan. El grupo de galáxidos (Familia Galaxiidae) es el más afectado, tanto por la gran depredación que sufren, como por verse desplazados de su nicho trófico en presencia de los salmónidos. La gestión de este problema ecológico es complicada debido al gran interés comercial y turístico que tienen los salmónidos en Chile, haciendo que las medidas gubernamentales para su eliminación sean escasas y de carácter puntual. Dentro de los impactos en los ecosistemas acuáticos Alonso y Castro-Díez (2015) revisan el estado de invasión en la península ibérica del caracol del cieno de Nueva Zelanda (Potamopyrgus antipodarum). Este pequeño caracol ha colonizado muchos ecosistemas acuáticos a lo largo del mundo, no estando la península ibérica libre del mismo. Su distribución ha ido aumentando a lo largo de los últimos años y su carácter invasor es ambiguo, siendo muy dependiente del ecosistema que invade. Entre sus impactos destaca su capacidad para cambiar la estructura de las comunidades de invertebrados, pudiendo alcanzar densidades de hasta 98300 individuos $/ \mathrm{m}^{2}$ en algunos ecosistemas invadidos. A pesar del potencial impacto de esta especie, aún no está claro cuáles son los mecanismos que facilitan su dispersión entre cuencas. En otro estudio Balmori et al. (2015) analizan las causas de la expansión y los impactos sobre las especies nativas del visón americano (Neovison vison) en España, que desde que se asilvestró en la década de los 60 del pasado siglo se ha expandido rápidamente por la cuenca del Duero. Además analizan las actuaciones necesarias para la conservación del visón europeo (Mustela lutreola), especie gravemente amenazada por su congénere americano. Esta misma especie exótica ha sido recientemente descubierta en la isla de Chiloé (Chile). Vergara y Valenzuela (2015) alertan en otro artículo de este monográfico del posible inicio de una invasión biológica en la isla y analizan los riesgos potenciales de este carnívoro en los ecosistemas insulares.
Desde una perspectiva más ecosistémica, dos revisiones de este monográfico analizan algunos de los mecanismos por los que las especies exóticas invasoras pueden alterar el funcionamiento de los ecosistemas. Lorenzo y Rodríguez-Echeverría (2015) recopilan la información científica sobre los impactos de las acacias australianas en el ecosistema edáfico. Estas especies causan alteraciones de la estructura de las comunidades microbianas edáficas, modificando los procesos y servicios de los ecosistemas invadidos. Entre sus impactos se encuentran la alteración de los ciclos biogeoquímicos del carbono y del nitrógeno. También causan una disminución de la disponibilidad hídrica en los ecosistemas invadidos. Además estas especies causan alteraciones de la diversidad, tanto funcional como genética, de las comunidades microbianas del suelo. Por su parte, Traveset (2015) se centra en la disrupción de las interacciones positivas (mutualismos), como mecanismo por el que algunas especies exóticas invasoras alteran la estructura y funcionamiento de los ecosistemas. Concretamente, se analizan casos en los que plantas, animales o microorganismos exóticos causan la alteración de tres tipos de mutualismos, polinización, dispersión y simbiosis entre plantas y microorganismos, así como sus consecuencias en la estructura y funcionamiento de las comunidades.

El artículo de Castro-Díez et al. (2015) aborda el problema metodológico que supone integrar medidas de impacto ecológico procedentes de estudios diversos. Dada la multiplicidad de parámetros que pueden ser utilizados para cuantificar impacto ecológico, los autores proponen agruparlos en categorías afines, en función de nivel de organización al que afecte (poblaciones, comunidades, ecosistemas) o del tipo de función ecosistémica que alteren. Dentro de cada categoría, la disparidad de magnitudes utilizadas se puede integrar en un único valor mediante técnicas matemáticas de metaanálisis. Los autores aplican su propuesta metodológica para evaluar los impactos causados por tres especies de árboles exóticos que invaden las riberas españolas.

Otros tres artículos del monográfico ponen el énfasis en la búsqueda de las causas que explican el éxito invasor. Roiloa et al. (2015) se centran en una propiedad compartida por muchas plantas exóticas invasoras, como es el crecimiento clonal. Estos autores analizan los mecanismos por los cuales esta estrategia de crecimiento contribuye a que las plantas exóticas tengan más probabilidades de establecerse, expandirse y causar impactos en los ecosistemas que alcanzan. Por otra parte, González-Moreno et al. (2015) analizan la vulnerabilidad de los ecosistemas a ser invadidos por especies exóticas, centrándose en la estructura del paisaje como factor que determina el grado de invasión. Estos autores muestran que las propiedades del paisaje son las que mejor explican el grado de invasión a escalas espaciales intermedias. También muestran que las alteraciones que el hombre causa en el paisaje favorecen el asentamiento de especies exóticas. Sin embargo, este efecto a menudo se manifiesta con cierto retardo, lo que dificulta la asociación entre la causa y la consecuencia. La importancia de la perturbación antrópica en la distribución de especies exóticas también se pone de manifiesto en el artículo de Cabra-Rivas et al. (2015). Este estudio diagnostica el estado de invasión de las riberas de tres ríos españoles por tres especies de árboles exóticos y analiza las propiedades de los hábitats más invadidos. En todos los casos los árboles invasores aparecen asociados a ambientes con alta disponibilidad de luz y elevada influencia antrópica.

Por último, con un enfoque más centrado en la gestión, Jiménez-Ruíz y Sánchez-Martínez (2015) analizan la eficiencia de diferentes técnicas de control y erradicación de la caña común (Arundo donax L.) en varios ecosistemas riparios de la península ibérica. Estos autores validan la eficacia de varios métodos que consiguen la eliminación del $100 \%$ de los ejemplares de esta especie tan dañina en los ambientes mediterráneos.

Con estos doce artículos esperamos contribuir a difundir el problema de las invasiones biológicas, a estimular el estudio de sus impactos -a menudo pobremente documentados- así como aportar directrices que lleven a una eficaz gestión de este, a menudo ignorado, vector de cambio global. 


\section{Agradecimientos}

Como editores invitados del presente monográfico queremos agradecer a todos los autores y revisores su contribución para que este monográfico haya salido adelante. También queremos agradecer a Luis Cayuela y Leyre Jiménez su labor de coordinación editorial. Este trabajo ha sido financiado por los siguientes proyectos de investigación: Ministerio de Economía y Competitividad (CGL2010-16388/BOS), Universidad de Alcalá (CCG2013/EXP054) y red REMEDINAL de la Comunidad de Madrid (S2009/AMB1783).

\section{Referencias}

Alonso, A., Castro Díez, P. 2015. El caracol acuático neozelandés del cieno (Potamopyrgus antipodarum): impactos ecológicos y distribución de esta especie exótica en la península ibérica. Ecosistemas 24(1): 52-58.

Andreu, J., Vila, M., Hulme, P.E. 2009. An assessment of stakeholder perceptions and management of noxious alien plants in Spain. Environmental Management 43:1244-1255.

Balmori, A., Santos, I., Carbonell, R. 2015. El visón americano Neovison vison (Schreber 1777) en España: posibles causas de su expansión e interacción con otros mamíferos semiacuáticos. Ecosistemas 24(1): 4-11.

Cabra-Rivas, I., Castro-Díez, P., Saldaña, A. 2015. Análisis de la invasión del hábitat ribereño por tres árboles exóticos en España. Ecosistemas 24(1): 18-28

Castro-Díez, P., Alonso, A., Gutiérrez-López, M., de las Heras, P., MedinaVillar, S., Pérez-Corona, E., Trigo, D., Vázquez de Aldana B.R. 2015. Integración de impactos ecológicos causados por plantas exóticas invasoras: propuesta metodológica. Ecosistemas 24(1): 12-17

Charles, H., Dukes, J.S. 2007. Impacts of invasive species on ecosystem services. En: Nentwig, W. (eds.), Biological Invasions, pp. 217-237. Springer-Verlag, Berlin-Heidelberg.
Elton, C.S. 1958. The ecology of invasions by animals and plants. Mathuen, London

García-Berthou, E., Almeida, D., Benejam, L., Magellan, K., Bae, M-J, Casals, F., Merciai, R. 2015. Impacto ecológico de los peces continentales introducidos en la Península Ibérica. Ecosistemas 24(1): 84-92.

González-Moreno, P., Delgado, J.D., Vilà, M. 2015. Una visión a escala de paisaje de las invasiones biológicas. Ecosistemas 24(1): 84-92.

Habit, E, González, J., Ortiz-Sandoval, J., Elgueta, A., Sobenes, C. 2015. Efectos de la invasión de peces en ríos y lagos de Chile. Ecosistemas 24(1): 43-51.

Jiménez-Ruíz, J., Sánchez-Martínez, F.J. 2015. I+D+i aplicada al control de especies invasoras en ecosistemas fluviales mediterráneos. El caso de la caña común (Arundo donax L.). Ecosistemas 24(1): 32-35.

Lorenzo, P., Rodríguez-Echeverría, S. 2015. Cambios provocados en el suelo por la invasión de acacias australianas. Ecosistemas 24(1): 59-66.

Mazza, G., Tricarico, E., Genovesi, P., Gherardi, F. 2014. Biological invaders are threats to human health: an overview. Ethology, Ecology and Evolution 26: 112-129

Pimentel, D. 2005. Environmental consequences and economic costs of alien species. En: Inderjit, S. (ed.), Invasive plants: Ecological and agricultural aspects, pp 269-276, Birkhäuser Basel. Springer. Verlag, Switzerland.

Roiloa, S.R., González Campoy, J., Retuerto, R. 2015. Importancia de la integración clonal en las invasiones biológicas. Ecosistemas 24(1): 76-83.

Traveset, A. 2015. Impacto de las especies exóticas sobre las comunidades mediado por interacciones mutualistas. Ecosistemas 24(1): 67-75.

Vergara, G., Valenzuela, J. 2015. Presencia de visón americano (Neovison vison, Schreber 1777) en Chiloé, Chile: ¿inicio de una invasión biológica? Ecosistemas 24(1): 29-31.

Vitousek, P.M., Walker, L.R. 1989. Biological invasion by Myrica faya in Hawaii: plant demography, nutrient fixation, ecosystem effects. Ecological Monographs 59:247-265. 